\title{
Análisis de la gestión de movilidad vehicular urbana utilizando Mapas Cognitivos Difusos
}

\section{Analysis of urban vehicle mobility management using Fuzzy Cognitive Maps}

\author{
Estefanía Rocha Tamayo \\ Universidad de Guayaquil \\ Guayaquil, Ecuador \\ estefania.rochat@ug.edu.ec
}

\author{
Jimmy Sornoza Moreira \\ Universidad de Guayaquil \\ Guayaquil, Ecuador \\ Jimmy.sornozam@ug.edu.ec \\ Orcid:0000-0002-0608-9216
Carlos Villarreal Vásquez
Universidad de Guayaquil
Guayaquil, Ecuador
carlos.villarrealv@ug.edu.ec

\author{
Lorenzo J. Cevallos Torres \\ Universidad de Guayaquil \\ Guayaquil, Ecuador \\ lorenzo.cevallost@ug.edu.ec \\ Orcid:0000-0002-7211-2891
}

\begin{abstract}
Resumen- El tráfico vehicular es un mal común en casi cualquier ciudad o país del mundo, lo cual afecta considerablemente a la población en general; para solventar esto se han utilizado modelos matemáticos y en este caso hemos considerado los mapas cognitivos difusos, los cuales son gráficos que se usan para representar causalidad entre diferentes factores o conceptos, los que inciden para solucionar un problema que no es sencillo de resolver. En el presente trabajo se implementa un modelo que permita hallar una solución al problema del tráfico vehicular aplicando la lógica difusa y mapas cognitivos difusos; su utilización permite incrementar la facilidad en la toma de decisiones. Se desarrolló un caso de estudio apoyado en una herramienta informática que facilita la realización de los mapas cognitivos difusos. Por medio del uso de mapas cognitivos difusos se obtendrá resultados que permiten analizar cuál es el impacto de los distintos elementos que afectan al tráfico vehicular, y determinar la factibilidad/eficacia de la información que se obtiene como producto final, la cual conduce al aporte de mejoras significativas de los servicios de transporte y seguridad vial.
\end{abstract}

Palabras Clave: Control de Tráfico vehicular, Toma de Decisiones, Mapas Cognitivos Difusos
Abstract - Vehicle traffic is a common problem in almost any city or country in the world, which considerably affects the general population. In order to solve this, mathematical models have been used, and in this case, we have considered fuzzy cognitive maps, which are graphs that are employed to represent causality between different factors or concepts that intervene to solve a problem that is not easy to solve. In this work, a model is implemented to allow finding a solution to the problem of vehicular traffic by applying fuzzy logic and fuzzy cognitive maps. Its use allows increasing ease in decision-making. A case study supported by a computer tool was developed to facilitate the elaboration of fuzzy cognitive maps. Through the use of fuzzy cognitive maps, results will be obtained to analyze the impact of the different elements that affect vehicle traffic and determine the feasibility/efficacy of the information obtained as a final product, which leads to the contribution of significant improvements in transportation and road safety services.

Keywords: Vehicle Traffic Control, Decision-Making, Fuzzy Cognitive Maps

Sumario: I Introducción, II Materiales y Métodos, III Caso de estudio: analizar tráfico con mapas cognitivos difusos, IV. Conclusiones.

Como citar: Rocha, Estefanía., Sornoza, Jimmy., Cevallos, Lorenzo., Villareal, Carlos. (2020). Análisis de la gestión de movilidad vehicular urbana utilizando Mapas Cognitivos Difusos. Revista Tecnológica - Espol, 32(1). Recuperado a partir de http://www.rte.espol.edu.ec/index.php/tecnologica/article/view/667 


\section{INTRODUCCIÓN}

A lo largo del tiempo, en las ciudades urbanas del mundo la fluidez vehicular ha aumentado considerablemente; esto se debe a factores culturales, económicos y sociales. La forma de desplazarse de un lugar a otro no solo afecta al conductor del automóvil sino también a los peatones y, aunque existen avances en las infraestructuras de carreteras en las ciudades, no se ha logrado resolver este problema [1]. Es importante realizar este estudio para conocer las inconformidades de la población debido al congestionamiento vehicular, y al mismo tiempo analizar los patrones que afectan al tráfico vehicular para poder encontrar una solución efectiva a esta problemática.

Se suele pensar que cuando existe un aumento en la infraestructura vial esto lleva a una mejora en la fluidez de vehículos; sin embargo, esto no es totalmente cierto, ya que hay otros factores que provocan el congestionamiento vehicular.

El mal diseño de las infraestructuras viales y la utilización de los controladores de tráfico que ya son obsoletos, y su falta de mantenimiento, son las causas principales que ocasionan que en varias ciudades del mundo tengan muchos problemas de transporte, por lo que cada vez se dan a conocer estrategias que ayudan a solventar este problema que afecta a muchas personas alrededor del mundo [2].

El tráfico vehicular es un mal común en muchas partes del mundo, por lo cual algunas personas han optado por crear soluciones que ayuden de manera positiva al congestionamiento vehicular; de esta manera Álvaro Ruiz de Somorcurio realizó una propuesta para el control de tráfico vehicular usando lógica difusa, en la cual desarrolló un módulo de control para el manejo de las variables usando algoritmo de lógica difusa por medio del Modelo Mandami, el cual permite reducir los tiempos de espera de conductores y una adecuada sincronización para que el flujo de automóviles sea más eficiente [3]. José Castán, Salvador Ibarra, Julio Laria, Javier Guzmán y Emilio Castán realizaron un estudio publicado el 15 de noviembre de 2014 [4] para poder controlar el tráfico, el mismo que estuvo basado en agentes inteligentes, y desarrollaron un modelo con el que manipulan las etapas del ciclo en una infraestructura del semáforo, en el cual usan la lógica difusa para el control multi agente de un semáforo basado en sensores inalámbricos, lo cual evitará accidentes y congestionamiento de carros. [4]

Con el fin de realizar un aporte significativo a la problemática de la congestión vehicular, se hace uso de Mapas Cognitivos Difusos definiendo los componentes que inciden en el congestionamiento de vehículos, lo cual permitirá realizar un análisis sobre los factores que afectan el problema antes mencionado, con el fin de obtener una solución.

Los mapas cognitivos difusos son grafos dirigidos, los nodos simbolizan conceptos, y las relaciones son influencias causales entre éstos. El peso de las relaciones muestra la influencia que tiene un nodo sobre el otro [5]. Las computadoras tienen la habilidad de realizar cálculos a grandes velocidades; por ende, son usadas cuando se requiere tomar una decisión de alta complejidad, y la lógica difusa brinda un medio para encapsular lo subjetivo de la toma de decisiones [6].

Una actividad importante en la tarea de toma de decisiones, sin duda alguna, es la construcción de los modelos que facilitarán la colaboración de expertos en los temas a analizar o problemas a resolverse, así como la agregación de modelos causales.

En el contexto de la toma de decisiones hay dos propiedades fundamentales: Aplicabilidad, determinada por su uso fácil, flexible y adaptable, es decir que sea práctica y, Fiabilidad, que está relacionada con las preferencias de la persona que toma las decisiones, y tener como respuesta un resultado cercano a las que darían los exportes [7].

Para desarrollar el Mapa Cognitivo Difuso se ha considerado usar la herramienta Mental Modeler, siendo el objetivo de este software brindar facilidad a los usuarios en la construcción de los modelos de MCD, y permitir flexibilidad para que los usuarios refinen y puedan comprobar sus modelos, brindando una planificación de gestión efectiva [8].

\section{A. Lógica Difusa}

\section{MATERIALES Y MÉTODOS}

La lógica difusa reconoce no simples valores falsos y verdaderos, sino valores con cierto grado de falsedad o veracidad; ésta proporciona un mecanismo de inferencia que ayuda a simular procesos de razonamiento humano en sistemas basados en el conocimiento [9]. La lógica difusa realiza aproximaciones matemáticas que ayudan a la resolución de un problema difícil de resolver.

Las variables lingüísticas brindan un medio de caracterización a fenómenos que son muy difíciles de darles una descripción cuantitativa, por ejemplo, cuando se habla de la verdad se dice que toma valores como muy verdadero, no muy cierto, falso, entre otros. Las relaciones causales por lo general son granulares, esto se representa por medio de variables lingüísticas [10], es decir, las variables lingüísticas permiten describir el estado de un objeto o fenómeno. Para entenderlo mejor se describe un ejemplo:

\section{Una variable numérica toma valores numéricos Estatura $=2$ (metros) \\ Una variable lingüística toma valores lingüísticos Estatura es alta}

Los valores lingüísticos forman un conjunto de etiquetas, por ejemplo: muy alta, medianamente alta, alta, baja, muy baja, medianamente baja y baja tal como se muestra en la Fig. 1.

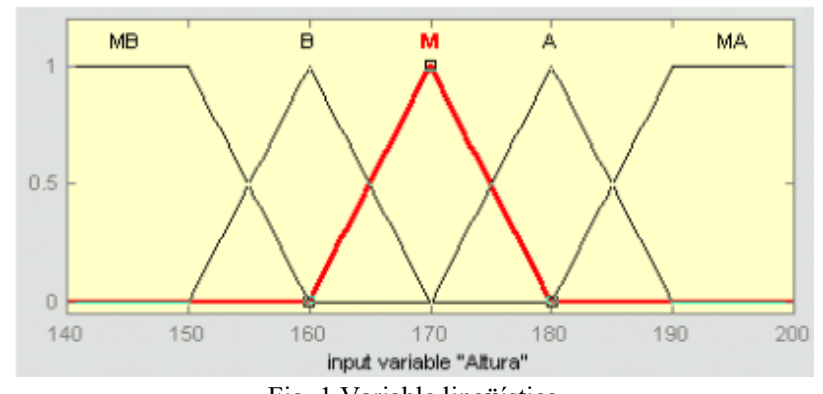

Fig. 1 Variable lingüística 
Las funciones de pertenencia sigmoidal son funciones continuas, además especifican un conjunto difuso normal y convexo tal como se ilustra en la Fig. 2.

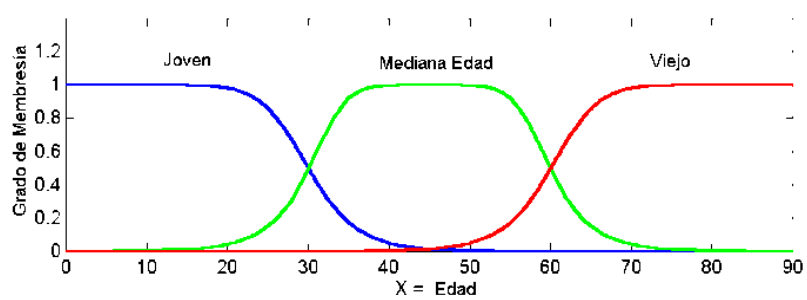

Fig. 2 Variable lingüística con función de pertenencia sigmoidal

Los conjuntos difusos son aquellos conjuntos que se usan para representar de manera matemática la indecisión en los distintos aspectos de la vida, brindando herramientas para utilizarlos [11]. Éstos se caracterizan por una función que asigna a cada elemento un grado de pertenencia oscilando entre menos uno y uno. Las nociones de unión, exclusión, intersección, relación, entre otras, se extienden a tales conjuntos y varias propiedades de estas nociones en el contexto de conjuntos difusos que se establecen [12].

La función de pertenencia $\mu \mathrm{A}$, para un conjunto difuso es definida de la siguiente forma:

$$
\mu \mathrm{Ci}(\mathrm{x}) \text { à }[0,1] \text { x E X }
$$

Esto representa el grado de pertenencia de la variable $\mathrm{x}$ en el conjunto difuso $\mathrm{Ci}$, que puede variar desde $0 \mathrm{a}+1$, y para definir en su totalidad el conjunto difuso $\mathrm{Ci}$, se debe definir $\mu \mathrm{Ci}(\mathrm{x})$ para todos los valores que pueda tomar $\mathrm{x}$ en el universo de X.

\section{B. Mapas Cognitivos Difusos}

En términos generales los conceptos de los mapas cognitivos difusos demuestran los factores claves y las características del sistema complejo modelado y representan: entradas, salidas, variables y tendencias de un sistema de modelado complejo [13].

Entre las ventajas de construir un mapa cognitivo difuso están las siguientes: la estructuración de un proceso, y construir el orden de todas las alternativas permitiendo un grado de causalidad por medio de un vector de pesos [14].

La causalidad es la representación de causa y efecto, su importancia se basa en que encuentra una explicación a los eventos del mundo real. Los mapas cognitivos difusos también son llamados mapas causales difusos, ya que realizan la representación de la causalidad que hay entre los componentes que lo conforman.

\section{Estructura de un Mapa Cognitivo Difuso}

Los mapas cognitivos difusos están compuestos de nodos, también llamados conceptos, los cuales representan variables. Los enlaces que hay entre estos conceptos son asignados con el signo + o - para representar si la relación es negativa o positiva entre los nodos; esto describe el grado de pertenencia que tienen los nodos. Éstos permiten crear y modelar sistemas enfocados en una explicación causal de interrelaciones entre los conceptos [15].
Al realizar la relación entre los conceptos se pueden establecer tres tipos de nodos: Conductores, los cuales son los nodos que no tienen nodos entrantes; Recibidores, aquellos nodos que tienen nodos entrantes y, Ordinarios, que son los nodos que tienen nodos entrantes y salientes.

Las direcciones de las relaciones son representadas por flechas tal como se ilustra en la Fig. 3, además permiten tener grados de causalidad entre un nodo al otro comprendido entre $-1 \mathrm{a}+1$.

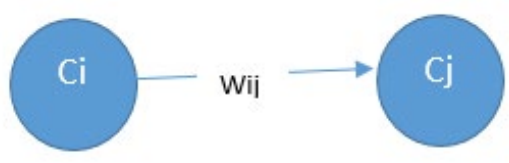

Fig. 3 Relaciones entre dos conceptos

La forma correcta de representar la causalidad es por medio de la matriz de adyacencia, en la cual se encuentran todas las relaciones que hay entre los nodos; se podrá observar el peso que tiene un nodo sobre otro.

De acuerdo a la TABLA I La matriz de adyacencia es una matriz cuadrada, utilizada con el fin de representar la conectividad que existe entre los nodos [16]. En la celda $\mathrm{Cij}$ se coloca el peso que tiene cada uno de los nodos con respecto a otro.

TABLA I

REPRESENTACIÓN DE UNA MATRIZ DE ADYACENCIA $\mathbf{C i} \quad \mathbf{C j}$

\begin{tabular}{lll}
$\mathrm{Ci}$ & Wij \\
$\mathrm{Cj}$ & $\mathrm{Wji}$ & \\
\hline
\end{tabular}

Las casillas que corresponden a la diagonal principal siempre tienen un peso de cero, debido a que no debería existir una relación entre sí mismo.

Hay tres tipos posibles relaciones causales entre nodos representados en la matriz:

- $\quad$ Wij $>0$, representa una causalidad positiva entre los nodos $\mathrm{Ci} \mathrm{y} \mathrm{Cj}$. El incremento o la disminución en el valor de $\mathrm{Ci}$ conduce al incremento o disminución en el valor de $\mathrm{Cj}$.

- $\mathrm{Wij}=0$, representa que no hay ninguna relación entre $\mathrm{Ci}$ y $\mathrm{Cj}$.

- $\quad$ Wij $<0$, representa una causalidad negativa entre los nodos $\mathrm{Ci} \mathrm{y} \mathrm{Cj}$. El incremento o la disminución en el valor de $\mathrm{Ci}$ conduce al incremento o disminución en el valor de $\mathrm{Cj}$.

Wij representa el peso de la relación que existe entre dos conceptos $\mathrm{Ci}$ y $\mathrm{Cj}$.

La simulación comienza por medio de la deducción del Mapa Cognitivo Difuso y de la definición del vector de estímulo. Se ha estimado usar la función sigmoidal para que los cambios de estado se realicen de manera continua y conocer el estado final en el que se encontrarán los nodos o conceptos que intervienen. La función sigmoidal es la siguiente.

$$
f(x)=(1 /(1+e-x)
$$

Fórmula 1. Función Sigmoidal 
La centralidad del grado se usa para encontrar el nodo más importante; éste se determina por medio de la suma del grado de entrada y del grado de salida, como se muestra en la siguiente fórmula:

$$
\begin{aligned}
& \mathrm{C}(\mathrm{v})=\mathrm{id}(\mathrm{v})+\mathrm{od}(\mathrm{v}) \\
& \text { Fórmula 2. Fórmula Centroide }
\end{aligned}
$$

La centralidad que existe en un nodo muestra qué tanto está relacionado un nodo con otro.

\section{Mental Modeler}

Es un software de modelamiento de datos que permite realizar análisis por medio de escenarios, tanto a individuos como a grupos de personas y les deja capturar sus conocimientos de manera estándar [17]. Se basa en Mapas Cognitivos Difuso, y se pueden crear de manera sencilla y rápida modelos de preocupaciones sociales, sistemas socio ecológicos o de medio ambiente.

Pasos para crear el modelamiento:

- Definir componentes.

- Definir relaciones entre componentes.

- Ejecutar escenarios "Qué pasa si" para saber la reacción del sistema según los rangos establecidos.

Modelo.- Es donde se definen los conceptos que se desean analizar y a la vez se coloca el grado de influencia entre un concepto a otro, realizando el mapa cognitivo difuso.

Matriz adyacente (Análisis estático)- Es una matriz de $\mathrm{n} \times \mathrm{m}$; para indicar el peso de la arista se coloca en la entrada del renglón i, columna j y reserva un valor especial null cuando indicamos una arista ausente. Representación en columnas y filas del conjunto de asignados a los conceptos de los mapas cognitivos difusos [18]. En Mental Modeler la matriz adyacente se llena automáticamente con los pesos que se definen en los modelos.

Métricas.- Se encuentran los valores de grados de entradas que es la suma de todos los pesos que ingresan a un concepto; valores de grados de salidas que es la suma de todos los pesos que salen de un nodo y, la centralidad que es la suma de los grados de entrada y salida.

Escenarios (Análisis dinámico).- El fin del análisis dinámico es realizar escenatrios hipotéticos que ayuden a simular el sitema de diferenes condiciones [19]. Mental Modeler permite realizar diferentes escenarios que permiten analizar los conceptos desde diferentes perspectivas.

\section{E. Movilidad urbana y siniestralidad vial}

En cuanto a la siniestralidad se mancomunan el factor humano y el diseño de las calles y avenidas. Ahora bien, por lo general en las carreteras la tipología común de siniestros en movilidad vehicular son las salidas de las vías, es decir, volcamiento, embestidas contra árboles, postes o columnas, derivados por los excesos de velocidad, alcohol e insomnio; en otros casos se incluyen los malos diseños y conservación de las vías [20].

En conjunto con lo antes expuesto, se señala el incumplimiento de las leyes y señales de tránsito que se ubican en diferentes partes de los urbanismos; debido a este atenuante es necesario recalcar a la ciudadanía las sanciones que corresponden a cada violación de las normativas de tránsito.

\section{F. Gestión de la movilidad vehicular}

El control de la gestión de movilidad vehicular es una parte fiundamental de la realización estratégica en el desarrollo de una ciudad urbana, obteniendo como fin conciliar la movilidad, el crecimiento y la competitivdad, tan necesarias hoy en día; para resolver el problema se debe contar con alta capacidad profesional y de liderazgo por parte de las autoridades urbanas [21].

En los últimos años se ha empezado a implementar políticas de gestión de movilidad vehicular; éstas incluyen cambios en la inversión en infraestructura de transporte, mayores impuestos a los vehìculos, cambios en el diseño de las calles, entre otras [22].

\section{CASO DE ESTUDIO: ANALIZAR TRÁFICO CON MAPAS COGNITIVOS DIFUSOS}

En nuestro caso de estudio, el problema de la congestión vehicular se va a considerar una propuesta en el cantón La Libertad- Provincia de Santa Elena-Ecuador, donde se encuentra claramente la problemática mencionada anteriormente. El agigantado crecimiento en la población y el sector productivo demandan a los gobiernos locales la indagación en relación a las soluciones más eficientes para los conflictos de movilidad del cantón La Libertad.

De acuerdo a la TABLA II se muestran los principales factores que afectan a la gestión del tráfico vehicular; se tendrán ocho variables o nodos: Semaforos dañados, Accidentes, Cantidad de Vehiculos, Vías en mal estado, Lluvias, Señales en buen estado, Trabajos en vías.

TABLA II

\begin{tabular}{|c|c|}
\hline Nodos & Descripción \\
\hline $\mathrm{C} 1$ & $\begin{array}{l}\text { Señales deTránsito en buen } \\
\text { estado. }\end{array}$ \\
\hline $\mathrm{C} 2$ & Semáforo en buen estado. \\
\hline $\mathrm{C} 3$ & Lluvia. \\
\hline $\mathrm{C} 4$ & Trabajos en las vías. \\
\hline $\mathrm{C} 5$ & Vías en mal estado. \\
\hline C6 & Congestionamiento vehicular. \\
\hline $\mathrm{C} 7$ & Accidente. \\
\hline $\mathrm{C} 8$ & Buen clima (No hay lluvias). \\
\hline
\end{tabular}

NODOS DEL MCD

Por medio de estos nodos se realizará un modelo de Mapa Cognitivo Difuso con sus relaciones y sus pesos tal como se ilustra en la Fig. 4.

Para poder efectuar la relación que hay entre un nodo y otro, hay que considerar dos preguntas:

1. ¿Cuando este componente incrementa, el otro componente incrementa o decrementa? 
2. ¿Éste incrementa o decrementa altamente, éste incrementa o decrementa mediamente o éste incrementa o decrementa bajamente.

También se debe considerar que los valores que se pueden colocar para indicar la influencia entre componentes y se encuentran en el rango de 1 al -1, para indicar el peso que tiene un nodo sobre otro

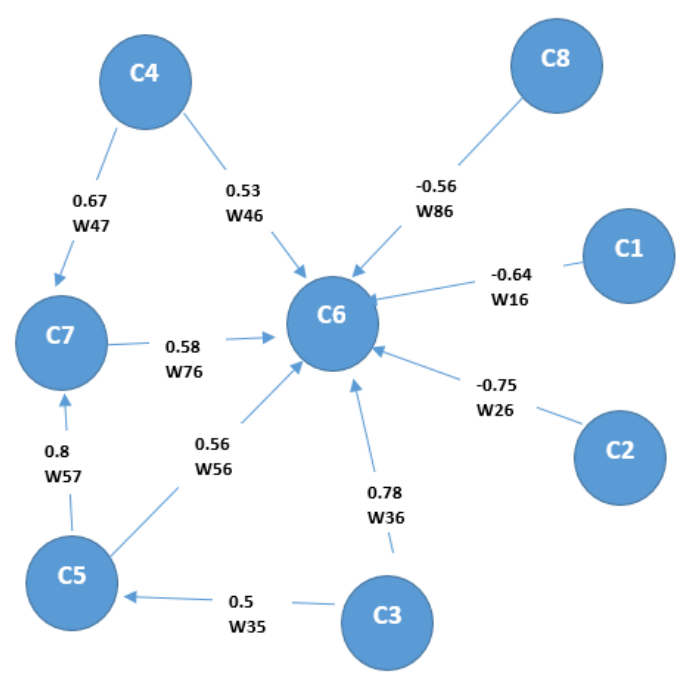

Fig. 4 Mapa Cognitivo Difuso del Tráfico Vehicular

Se realizará la lectura de un nodo para comprender cómo se lee el modelo:

$\mathrm{Si}$ los accidentes incrementan (C7), la congestión vehicular incrementa (C6).

De esta manera se realizará la lectura de cada nodo o concepto de este modelo, para mayor comprensión.

Para identificar el peso de cada nodo, se consulta a especialistas en el tema, se realizan preguntas que ayuden a identificar los factores que inciden en el problema, por lo cual un experto en el área de gestión vehicular ayudó a realizar el mapa cognitivo difuso con los diferentes pesos de cada nodo. [23]

De acuerdo a la TABLA III en donde se considera la variable lingüística Cantidad, con sus respectivas etiquetas linguisticas, las cuales ayudarán a darle un valor y sentido a la variable de estudio.

TABLA III ETIQUETA LINGÜÍSTICA

\begin{tabular}{ll} 
Etiquetas Lingüísticas & Valor \\
\hline Demasiado & 1 \\
Medianamente mucho & 0.5 \\
Mucho & 0.25 \\
& 0 \\
Poco & -0.25 \\
Mediamante Poco & -0.50 \\
Muy poco & -1
\end{tabular}

Las relaciones que existen en cada nodo, se deben detallar en la matriz de adyacencia, indicando el peso de cada nodo tal como se muestra en la TABLA IV.
TABLA IV

CASO DE ESTUDIO: REPRESENTACIÓN DE MATRIZ DE ADYACENCIA

\begin{tabular}{lllllllll}
\hline & C1 & C2 & C3 & C4 & C5 & C6 & C7 & C8 \\
\hline C1 & W11 & W12 & W13 & W14 & W15 & W16 & W17 & W18 \\
C2 & W21 & W22 & W23 & W24 & W25 & W26 & W27 & W28 \\
C3 & W31 & W32 & W33 & W34 & W35 & W36 & W37 & W38 \\
C4 & W41 & W42 & W43 & W44 & W45 & W46 & W47 & W48 \\
C5 & W51 & W52 & W53 & W54 & W55 & W56 & W57 & W58 \\
C6 & W61 & W62 & W63 & W64 & W65 & W66 & W67 & W68 \\
C7 & W71 & W72 & W73 & W74 & W75 & W76 & W77 & W78 \\
C8 & W81 & W82 & W83 & W84 & W85 & W86 & W87 & W88 \\
\hline
\end{tabular}

La matriz de adyacencia es una matriz que muestra los pesos que tienen entre un nodo a otro. La matriz es muy sencilla de realizar, solo se escriben los pesos que tiene un nodo sobre otro nodo, los cuales fueron definidos por el experto en el tema.

Una vez obtenida la matriz de adyacencia, se necesita establecer los patrones de entrada del llamado vector de entrada, para poder simular los escenarios que se necesite analizar.

El vector de entrada es el siguiente:

$$
\begin{aligned}
& \mathrm{E}=\left[\begin{array}{llllllll}
\mathrm{E} 1 & \mathrm{E} 2 & \mathrm{E} 3 & \mathrm{E} 4 & \mathrm{E} 5 & \mathrm{E} 6 & \mathrm{E} 7 & \mathrm{E} 8
\end{array}\right] \\
& \mathrm{E}=\left[\begin{array}{llllllll}
0 & 0 & 0 & 0 & 1 & 0 & 0 & 0
\end{array}\right]
\end{aligned}
$$

Cero significa que el nodo está apagado y 1 que el nodo está prendido; también se pueden usar valores compredidos desde -1 hasta 1 , y en este caso se encenderá E5 y se le coloca un valor muy alto; el resto de los nodos quedan apagados.

De acuerdo a la TABLA V, los valores del vector de entrada dependerán de lo que se quiera analizar en determinado momento.

TABLA V

MULTIPLICACIÓN DEL VECTOR DE ENTRADA CON MATRIZ ADYACENCIA

\begin{tabular}{lllllllll}
\hline & C1 & C2 & C3 & C4 & C5 & C6 & C7 & C8 \\
\hline C1 & W11 & W12 & W13 & W14 & W15 & W16 & W17 & W18 \\
C2 & W21 & W22 & W23 & W24 & W25 & W26 & W27 & W28 \\
C3 & W31 & W32 & W33 & W34 & W35 & W66 & W37 & W38 \\
C4 & W41 & W42 & W43 & W44 & W45 & W46 & W47 & W48 \\
C5 & W51 & W52 & W53 & W54 & W55 & W56 & W57 & W58 \\
C6 & W61 & W62 & W63 & W64 & W65 & W66 & W67 & W68 \\
C7 & W71 & W72 & W73 & W74 & W75 & W76 & W77 & W78 \\
C8 & W81 & W82 & W83 & W84 & W85 & W86 & W87 & W88
\end{tabular}

$\mathrm{VR}=[\mathrm{E} 1 \mathrm{E} 2 \mathrm{E} 3 \mathrm{E} 4 \mathrm{E} 5 \mathrm{E} 6 \mathrm{E} 7 \mathrm{E} 8]$ 
Se debe realizar la multiplicación del vector con la matriz de adyacencia, de la cual obtenemos un vector resultante:

\section{$\mathrm{VR} 1=[\mathrm{R} 1 \mathrm{R} 2 \mathrm{R} 3 \mathrm{R} 4 \mathrm{R} 5 \mathrm{R} 6 \mathrm{R} 7 \mathrm{R} 8]$}

Aplicando la fórmula 1 , donde $\mathrm{x}$ es cada elemento del vector resultante, se aplica esta función en todos los elementos del vector resultante VR de la multiplicación, obteniendo un nuevo vector resultado ya habiendo aplicado la función sigmoidal.

\section{$\mathrm{VS}=[\mathrm{S} 1 \mathrm{~S} 2 \mathrm{~S} 3 \mathrm{~S} 4 \mathrm{~S} 5 \mathrm{~S} 6 \mathrm{~S} 7 \mathrm{~S} 8]$}

El vector resultante VS, será el nuevo vector de entrada en la siguiente iteración; se realiza este procedimiento para los vectores resultantes VS en cada iteración, y este proceso continúa hasta cuando los valores de entradas y los valores de salida sean los mismo. Ver el resultado en la tabla 8 .

\section{RESULTADOS}

Es preciso dar a conocer cuáles han sido los resultados obtenidos en esta investigación con el propósito de destacar la solución que se le ha dado al problema por medio del análisis de la información. Aunque no es nuestro enfoque principal, se usó Big Data para analizar la información del tráfico permitiendo realizar consultas dependiendo de las necesidades de los analistas. Sin embargo, para dar solución al problema del tráfico se usaron Mapas Cognitivos Difusos.

Una vez construido el MCD, en la TABLA VI se realiza la matriz adyacente: se colocan todos los conceptos tanto en filas como en columnas (cabecera) y se ubican los pesos de cada nodo que fueron definidos en el modelo en su respectiva celda.

TABLA VI RESULTADO DE MATRIZ DE ADYACENCIA

\begin{tabular}{|c|c|c|c|c|c|c|c|c|}
\hline & C1 & $\mathrm{C} 2$ & C3 & $\mathrm{C} 4$ & C5 & C6 & C7 & C8 \\
\hline $\mathrm{C} 1$ & 0 & 0 & 0 & 0 & 0 & -0.64 & 0 & 0 \\
\hline $\mathrm{C} 2$ & 0 & 0 & 0 & 0 & 0 & -0.75 & 0 & 0 \\
\hline $\mathrm{C} 3$ & 0 & 0 & 0 & 0 & 0.5 & 0.78 & 0 & 0 \\
\hline $\mathrm{C} 4$ & 0 & 0 & 0 & 0 & 0 & 0.53 & 0.67 & 0 \\
\hline C5 & 0 & 0 & 0 & 0 & 0 & 0.56 & 0.8 & 0 \\
\hline C6 & 0 & 0 & 0 & 0 & 0 & 0 & 0 & 0 \\
\hline C7 & 0 & 0 & 0 & 0 & 0 & 0.58 & 0 & 0 \\
\hline C8 & 0 & 0 & 0 & 0 & 0 & $-0-56$ & 0 & 0 \\
\hline
\end{tabular}

En la TABLA VII Se debe evaluar la centralidad de un nodo para conocer cuál es el nodo más importante; éste se lo encuentra por medio de las entradas y salidas de un concepto. Para hallar las entradas de un concepto se deben sumar todos los valores entrantes de dicho nodo; para hallar las salidas de un concepto se deben sumar todos los valores salientes de dicho nodo. Se calcula la centralidad usando la fórmula 2.

TABLA VII

RESULTADO DE LA CENTRALIDAD DE LOS NODOS

\begin{tabular}{cccc} 
Nodo & Entradas & Salidas & Centralidad \\
\hline C1 & 0 & 0.64 & 0.64 \\
C2 & 0 & 0.75 & 0.75 \\
C3 & 0 & 1.28 & 1.28 \\
C4 & 0 & 1.20 & 1.20 \\
C5 & 0.5 & 1,36 & 1.86 \\
C6 & 4.4 & 0 & 4.4 \\
C7 & 1.47 & 0.58 & 2.05 \\
C8 & 0 & 0.56 & 0.56 \\
\hline
\end{tabular}

El número de iteraciones no es definido, ni por la cantidad de conceptos ni por los pesos, sino que se realiza el proceso explicado en el punto 3 para hallar el vector de salida de cada iteracción, y éste se repite hasta que el vector de salida sea igual que el vector de entrada como se visualiza en la TABLA VIII.

TABLA VIII

ITERACIONES DEL MCD

\begin{tabular}{|c|c|c|}
\hline Iteración & Vector entrada & Vector de salida \\
\hline 1 & $0,0,0,0,1,0,0,0$ & $\begin{array}{l}0.5,0.5,0.5,0.5,0.5,0.64, \\
0.69,0.50\end{array}$ \\
\hline 2 & $\begin{array}{l}0.5,0.5,0.5,0.5,0.5,0.64,0.69, \\
0.50\end{array}$ & $\begin{array}{llll}0.5,0.5,0.5,0.5, & 0.56, & 0.59, \\
0.68,0.50 & \end{array}$ \\
\hline 3 & $\begin{array}{l}0.5,0.5,0.5,0.5,0.56,0.59 \\
0.68,0.50\end{array}$ & $\begin{array}{lll}0.5,0.5,0.5,0.5, & 0.56,0.60, \\
0.69,0.50 & \end{array}$ \\
\hline 4 & $\begin{array}{l}0.5,0.5,0.5,0.5,0.56,0.60 \\
0.69,0.50\end{array}$ & $\begin{array}{lll}0.5,0.5,0.5,0.5, & 0.56,0.60, \\
0.69,0.50 & \end{array}$ \\
\hline
\end{tabular}

\section{A. Uso de la herramienta Mental Modeler}

En la Fig. 5 Mental Modeler permite modelar de manera ágil el MCD, agregando los componentes necesarios que se necesitan para resolver este problema, añadiéndoles el peso a cada componente.

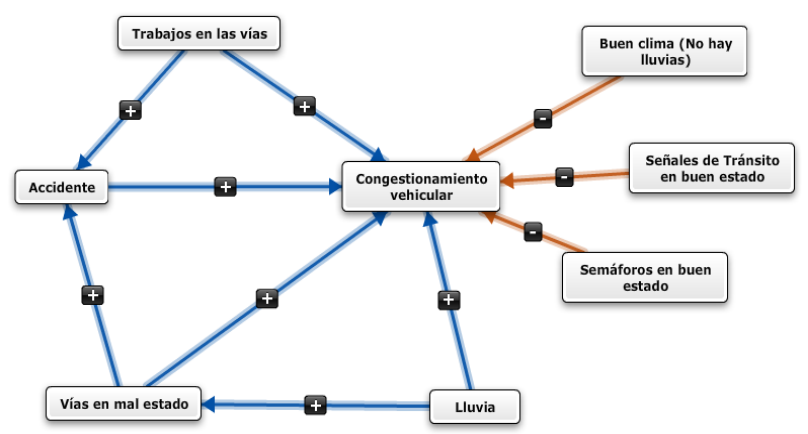

Fig. 5 Mental Modeler: Mapa Cognitivo Difuso

En las Fig. 6 y Fig. 7, la herramienta llena automáticamente los valores en la matriz cuadrada dependiendo del peso que se colocó al realizar el modelo, es decir facilita la labor de estar llenando celda por celda. 


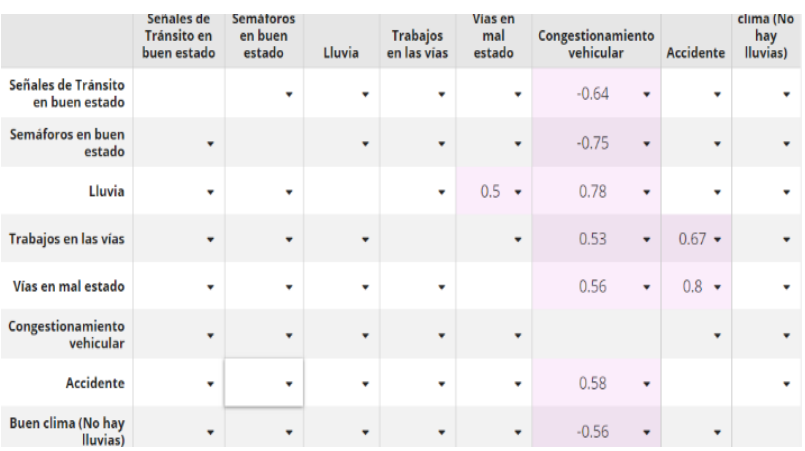

Fig. 6 Mental Modeler: Resultado de Matriz de adyacencia

Mental Modeler coloca los valores de entrada y salida de cada nodo y calcula la centralidad que posee cada nodo, optimizando tiempo al usuario, ya que lo realiza de manera automática.

\begin{tabular}{|c|c|c|c|c|c|}
\hline Component & Indegree & Outdegree & Centrality & $e^{\cdot}$ & Type $\mathbf{T}$ \\
\hline $\begin{array}{l}\text { Señales de Tránsito } \\
\text { en buen estado }\end{array}$ & 0 & 0.64 & 0.64 & . & driver \\
\hline $\begin{array}{l}\text { Semáforos en buen } \\
\text { estado }\end{array}$ & 0 & 0.75 & 0.75 & . & driver \\
\hline Lluvia & 0 & 1.28 & 1.28 & 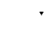 & driver \\
\hline Trabajos en las vías & 0 & 1.2000000000000002 & 1.2000000000000002 & 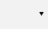 & driver \\
\hline Vias en mal estado & 0.5 & 1.36 & 1.86 & . & ordinary \\
\hline $\begin{array}{l}\text { Congestionamiento } \\
\text { vehicular }\end{array}$ & 4.4 & 0 & 4.4 & & receiver \\
\hline Accidente & 1.4700000000000002 & 0.58 & 2.0500000000000003 & . & ordinary \\
\hline $\begin{array}{l}\text { Buen clima (No hay } \\
\text { lluvias) }\end{array}$ & 0 & 0.56 & 0.56 & . & driver \\
\hline
\end{tabular}

Fig. 7 Mental Modeler: Resultado de la centralidad de los nodos

En la Fig. 8 se ingresa el vector de entrada, es decir qué conceptos se considera encender para conocer en cuánto influye al resto de los elementos; en este caso se enciende el nodo Vías en mal estado.

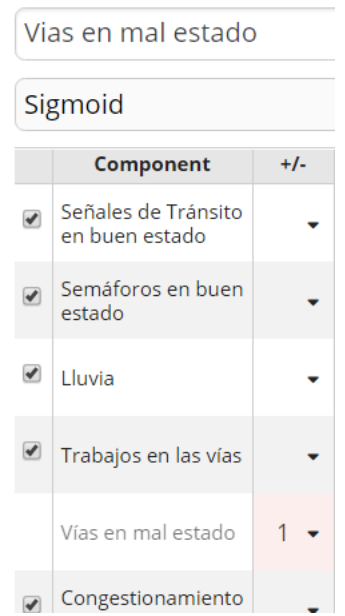

Fig. 8 Mental Modeler: Vector de entrada

Como se visualiza en la Fig. 9, la herramienta permite visualizar qué impacto tiene el nodo Vías en mal estado en el modelo, por lo cual se ve afectado el Congestionamiento vehicular y los Accidentes. Como se observa en la figura 4, Vías en mal estado está directamente relacionado con los conceptos mencionados anteriormente, por ende, se ven seriamente comprometidos.

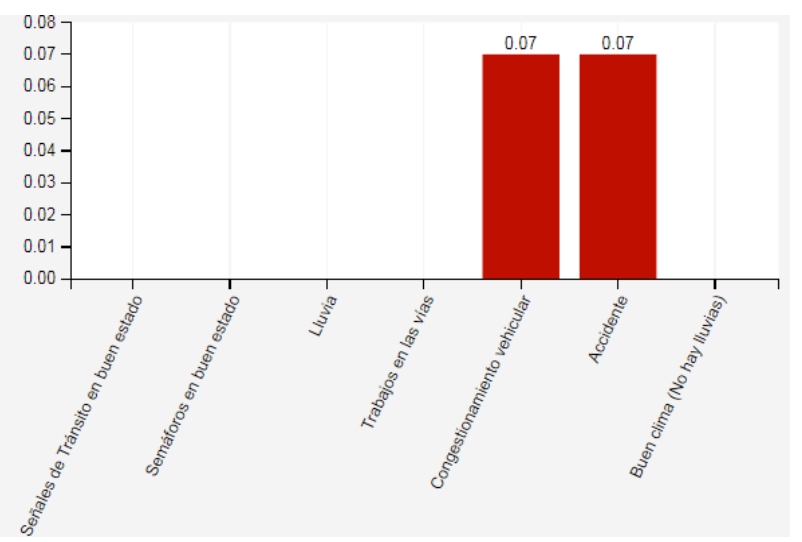

Fig. 9 Resultado de escenario en Mental Modeler

\section{CONCLUSIONES}

El uso de mapas cognitivos difusos permitió identificar la situación actual con respecto al tráfico vehicular ya que, con ayuda de expertos en el área de gestión vehicular, se logró obtener los factores que conllevan al congestionamiento de carros.

Se logró realizar un modelo eficientemente estructurado lo cual permitió examinar cómo afectan un factor en relación con otro, y así conocer cuál es el factor más relevante en este estudio, para su posterior análisis. El estudio de caso demostró la factibilidad del procedimiento y servirá de gran aporte sobre todo para ayudar a las partes interesadas y las personas encargadas de las tomas de decisiones a dar sentido a una situación compleja y negociar soluciones; se analizó de manera pertinente cada uno de los factores que inciden en este problema.

Al realizar el MCD, se pudo obtener las siguientes ventajas competitivas: permite examinar las relaciones que hay entre conceptos, integrar el conocimiento de expertos en el tema y realizar análisis dinámico; es decir, simular diferentes escenarios según la situación que se quiera considerar en determinado momento.

\section{REFERENCIAS}

[1] Pedraza, Luis Fernando, Hernández, César Augusto y López, Danilo Alfonso. Control de tráfico vehicular usando ANFIS. Bogotá : s.n., 2012.

[2] Espinoza, F., y otros. Machine vision algorithms applied to dynamic traffic light control. 2013.

[3] Ruiz de Somocurcio Salas, Alvaro Enrique. Control de tráfico vehicular utilizando logica difusa. Lima : s.n., 2008.

[4] Castán, José A., y otros. Control de tráfico basado en agentes inteligentes. 2014.

[5] Contreras, Juan. Modelamiento realístico de sistemas dinámicos complejos mediante mapas cognitivos difusos. 2016.

[6] Hazelzet, Jan A. Can fuzzy logic make things more clear? 2009.

[7] Singh, A. Architecture value mapping using fuzzy cognitive maps as a reasoning mechamism for multi-criteria conceptual design evaluation. Missouri : s.n., 2011.

[8] Gray, Steven A., y otros. Mental Modeler: A Fuzzy-Logic Cognitive Mapping Modeling Tool for Adaptive Enviromental Management. Hawaii : s.n., 2013.

[9] Morcillo, Carlos González. Lógica Difusa Una introducción práctica. 2013.

[10] Garcia-Cascales, M. y Lamata, M. Nueva aproximación al método topsis difuso con etiquetas linguísticas. 2010.

[11] Rodríguez, Mónica y Huertas, Yennifer. Metodología para el diseño de conjuntos difusos a partir de opiniones de expertos. 2016.

[12] Zadeh, L. A. Information and control. California : s.n., 1965. 
[13] Groumpos, Peter. Fuzzy Cognitive Maps: Basic Theores and Their Application to Complex System. Berlin : s.n., 2010.

[14] Leyva Vásquez, Maikel, y otros. Modelo para el análisis de escenarios basados en mapas cognitivos difusos estudio de caso en software biomédico. Bogotá : s.n., 2012.

[15] Papageorgiou, E. I. y Stylios, C. D. Fuzzy cognitive maps: Basic theories and their application to complex systems. Berlin : s.n., 2008.

[16] Salmeron, Jose L., Vidal, Rosario y Mena, Angel. Ranking fuzzy cognitive map based scenarios with Topsis. 2012.

[17] Mental Modeler Org. Mental Modeler. [En línea] 2015. http://www.mentalmodeler.org/.

[18] Peréz Teruel, Karina. Modelo de proceso de logro de concenso de mapas cognitivos difusos para la toma de decisiones en grupo. Habana : s.n., 2014.

[19] Stach, Wojciech. Leraning and Agregation of Fuzzy Cognitive Maps- An Evolutionary Approach. Alberta : s.n., 2010.

[20] Pérez, Fernando, y otros. Analysis of vehicular traffic flow using a macroscopic model. Puebla : s.n., 2013.

[21] Bill, Alberto. Congestión de Tránsito El problema y cómo enfrentarlo. s.l. : Santiago de Chile, 2003.

[22] Todd, Litman. Gestión de la movilidad para México. Cuauhtémoc : s.n., 2012.

[23] Leyva Vásquez, Maikel, y otros. Mapas cognitivos difusos para la selección de proyectos de tecnologías de la información. 2013. 